\title{
Influence of Structural and Dielectric Anisotropy on the Dielectrophoresis of Single-Walled Carbon Nanotubes
}

\section{Supporting Information}

Sabine Blatt, ${ }^{* 1}$ Frank Hennrich, ${ }^{1}$ Hilbert v. Löhneysen, ${ }^{2,3}$ Manfred M. Kappes, ${ }^{1,4}$ Aravind Vijayaraghavan $^{1}$ and Ralph Krupke ${ }^{*, 1,2}$

${ }^{1}$ Institut für Nanotechnologie, Forschungszentrum Karlsruhe, D-76021 Karlsruhe, Germany

${ }^{2}$ Physikalisches Institut, Universität Karlsruhe, D-76128 Karlsruhe, Germany

${ }^{3}$ Institut für Festkörperphysik, Forschungszentrum Karlsruhe, D-76021 Karlsruhe, Germany

${ }^{4}$ Institut für Physikalische Chemie, Universität Karlsruhe, D-76128 Karlsruhe, Germany

*Corresponding authors: blatt@int.fzk.de, krupke@int.fzk.de 


\section{Preparation of nanotube dispersions}

The single-walled carbon nanotubes (SWNTs) used in our experiments were grown by pulsed laser vaporization with $\mathrm{Ni}$ and $\mathrm{Co}$ as catalyst. To obtain well dispersed nanotube suspensions, the raw material was sonicated in $\mathrm{D}_{2} \mathrm{O}$ with 1 weight $\%$ of sodium cholate $(\mathrm{NaCh})$ as surfactant (also sodium dodecylbenzene sulfonate (SDBS) and Tris cholate) and centrifuged. ${ }^{1}$ The suspension was then separated into different length fractions by size-exclusion chromatography (typical length $500-1000$ $\mathrm{nm}){ }^{2}$ By comparing the absorption spectra of the nanotube suspensions (see Supplementary Figure 1) with a reference spectrum of a suspension with known concentration, we determined the concentration of the suspensions to be $\sim 100 \mu \mathrm{g} / \mathrm{ml}$.

\section{Additional measurements}

The polarization dependent absorption measurements were repeated for samples prepared with SWNTs diluted in $\mathrm{D}_{2} \mathrm{O}$ with $1 \mathrm{wt} \%$ SDBS (sodium dodecylbenzene sulfonate). Supplementary Figures 3 - 6 show the spectra of length separated or centrifuged nanotubes, deposited with frequencies $v=10-80 \mathrm{MHz}$ and voltages $V_{\mathrm{pp}}=40-80 \mathrm{~V}$.

Additionally, Raman spectroscopy was done with a Dilor XY triple monochromator and an $\mathrm{Ar}-\mathrm{Kr}$ laser for excitation. The spectra were recorded for two excitation laser lines, 514.5 and $647.1 \mathrm{~nm}$. To study the polarization dependence, we used a $\lambda / 2$-plate to rotate the polarization of the incident light with respect to the fixed orientation of the sample. The measurements were repeated for different spots on the sample.

A comparison of the Raman spectra measured between the electrodes for the two laser energies shows that the degree of alignment is much higher in the case of metallic nanotubes than for semiconducting nanotubes (Supplementary Figures 8 and 7). The axis of preferential orientation is parallel to the electric field during deposition. The spectra measured above the electrodes show equal intensity for parallel and perpendicular polarization. 


\section{Numerical simulations}

The finite-element calculations are based on the quasi-electrostatic form of the Maxwell equations with the potential phasor being entirely real. ${ }^{3}$ The potential $\Phi$ is then given by the Laplace equation, $\nabla(\varepsilon \nabla \Phi)=0$, where $\varepsilon$ is the permittivity of the surfactant medium. According to our impedance measurements the capacitive component originating from the double layer diminishes for frequencies. $v>10^{5} \mathrm{~Hz}$ For the frequencies used in our experiments we can thus neglect the influence of ionic double layers at the electrode surfaces and set the effective electrode potential equal to the applied potential $\left(\Phi_{0}= \pm V_{\mathrm{pp}} / 2\right)$.

The array of interdigitated electrodes consists of 140 electrode fingers with a length of $\sim 360 \mu \mathrm{m}$ and a width of $1.8 \mu \mathrm{m}$ each, separated by $1.8 \mu \mathrm{m}$ wide gaps. The thickness of the Al layer is $140 \mathrm{~nm}$ and that of the quartz glass is $0.5 \mathrm{~mm}$. As the extension in the y-direction, i.e. parallel to the fingers, is much larger than the finger and gap width, being the relevant dimension in the x-direction, we can reduce the three-dimensional problem to a two-dimensional one. The properties of the nanotubes and the suspension are used as given in the main text.

\section{References}

(1) O’Connell, M. J.; Bachilo, S. M.; Huffmann, C. B.; Moore, V. C.; Strano, M. S.; Haroz, E. H.; Railon, K. L.; Boul, P. J.; Noon, W. H.; Kittrell, C.; Ma, J.; Hauge, R. H.; Weisman, R. B.; Smalley, R. E. Science 2002, 297, 593-596.

(2) Hennrich, F.; Krupke, R.; Arnold, K.; Rojas Stutz, J. A.; Lebedkin, S.; Koch, Th.; Schimmel, Th.; Kappes, M. M. J. Phys. Chem. B 2007, 111, 1932-1937.

(3) Marquardt, C. W.; Blatt, S.; Hennrich, F.; v. Löhneysen, H.; Krupke, R. App. Phys. Lett. 2006, $89,183117$. 


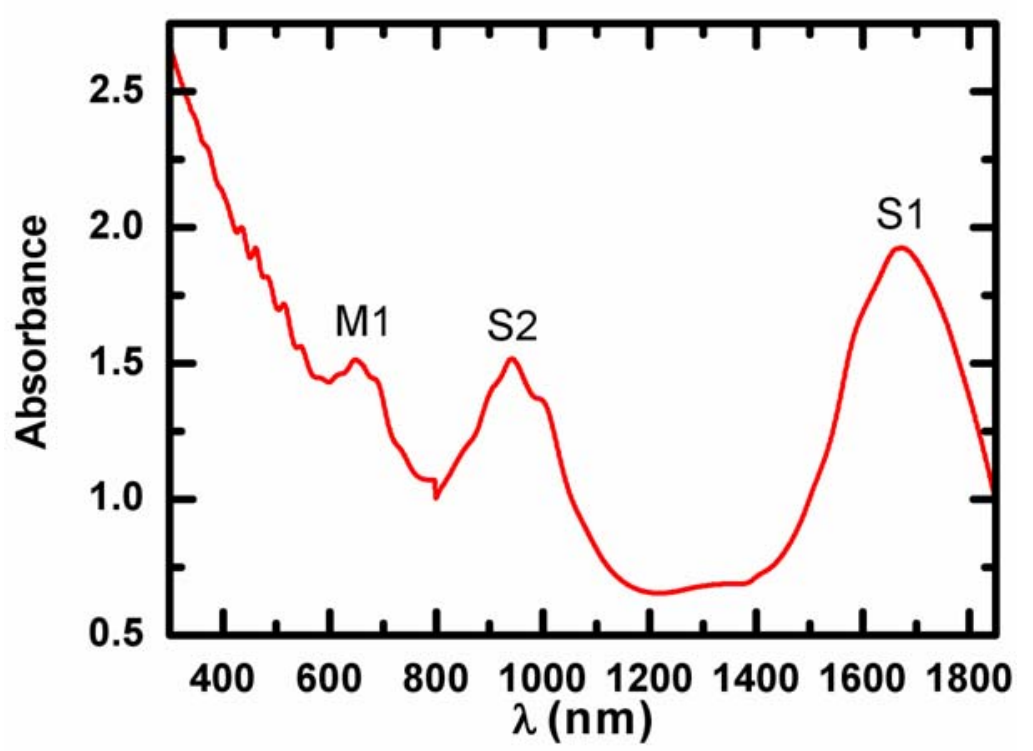

Supplementary Figure 1. Optical absorption spectrum of length separated SWNTs dispersed in $\mathrm{D}_{2} \mathrm{O}$ with 1 weight $\%$ sodium cholate. The SWNT concentration is $\sim 100 \mu \mathrm{g} / \mathrm{ml}$. 


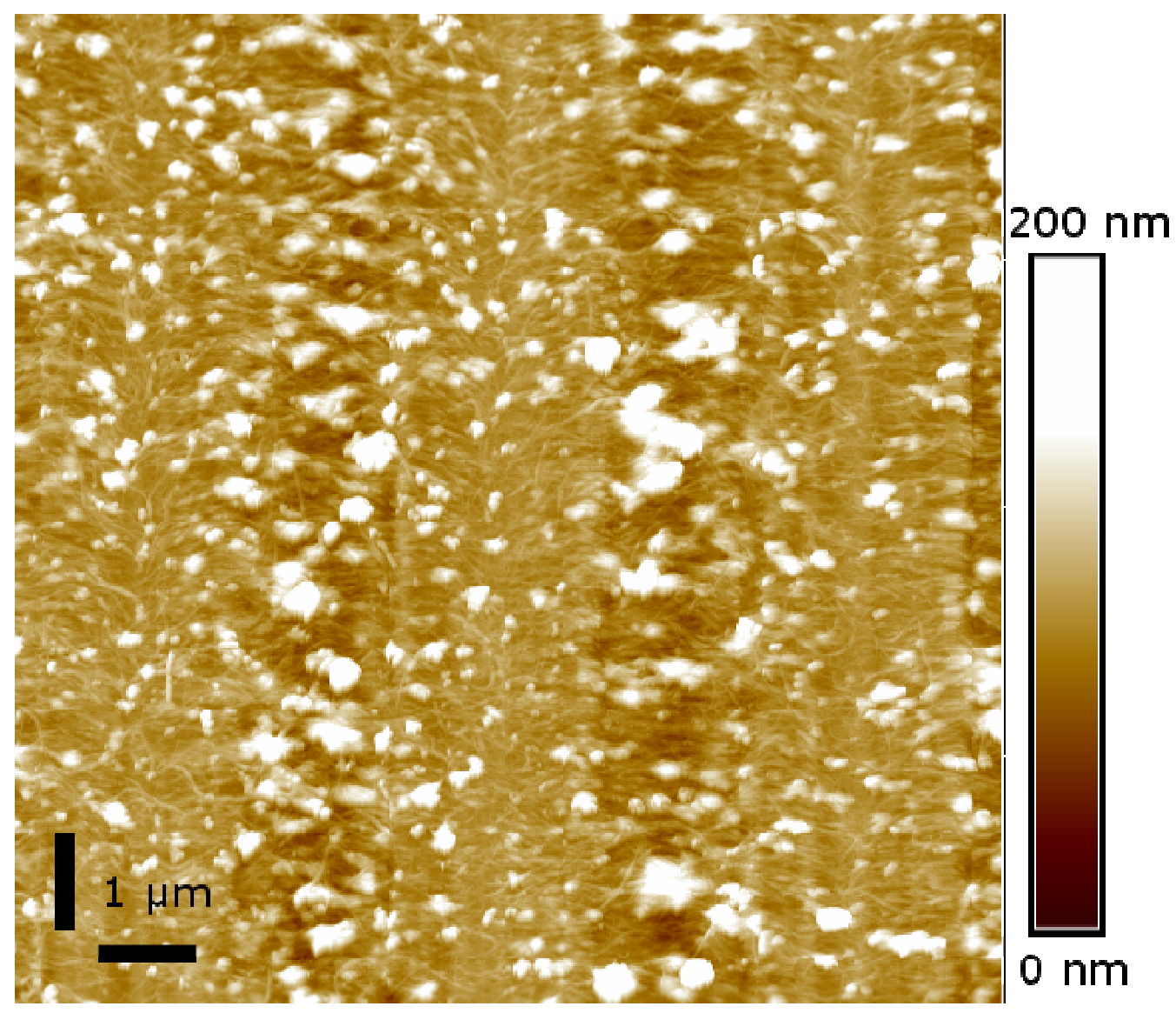

Supplementary Figure 2. Atomic force microscope image of a typical sample of nanotubes deposited onto an array of interdigitated electrodes. The areas with light brown background correspond to three electrodes, the darker background marks the gaps. The thickness of the nanotube film was determined to $\sim 60 \mathrm{~nm}$. 


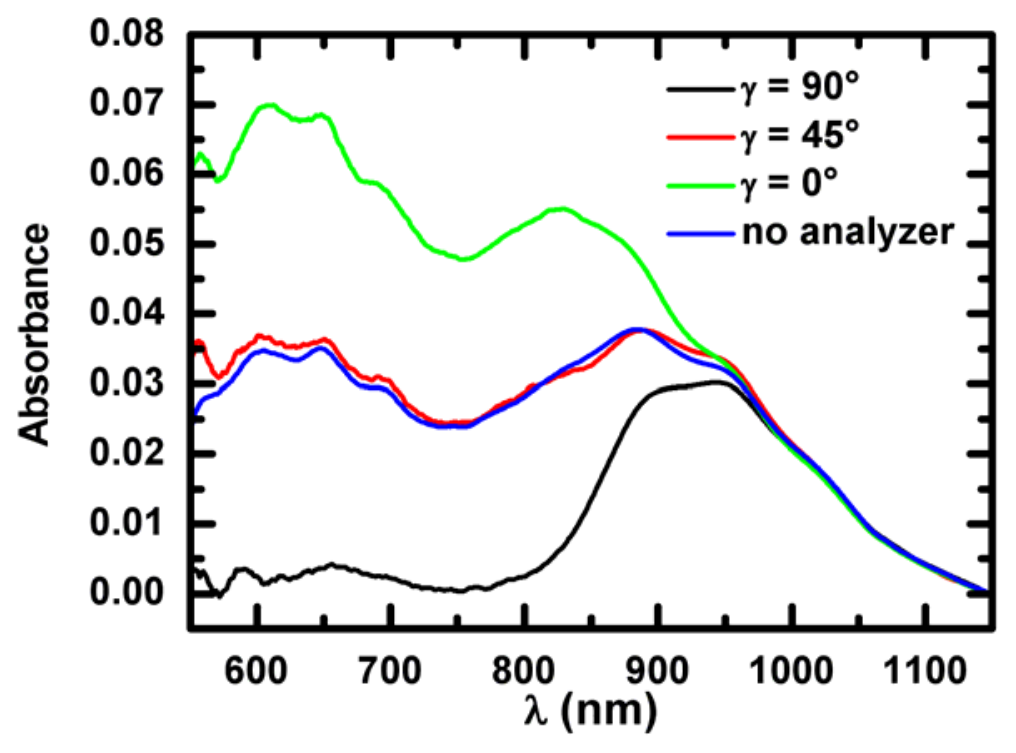

Supplementary Figure 3. Polarization dependent absorption spectra of length separated SWNTs in $1 \mathrm{w} \%$ SDBS, deposited with $v=10 \mathrm{MHz}$ and $V_{\mathrm{pp}}=40 \mathrm{~V}$. The raw data was smoothed and a linear background was subtracted. Metallic and small diameter semiconducting nanotubes are well aligned parallel to the electric field, while semiconducting nanotubes are randomly oriented. 


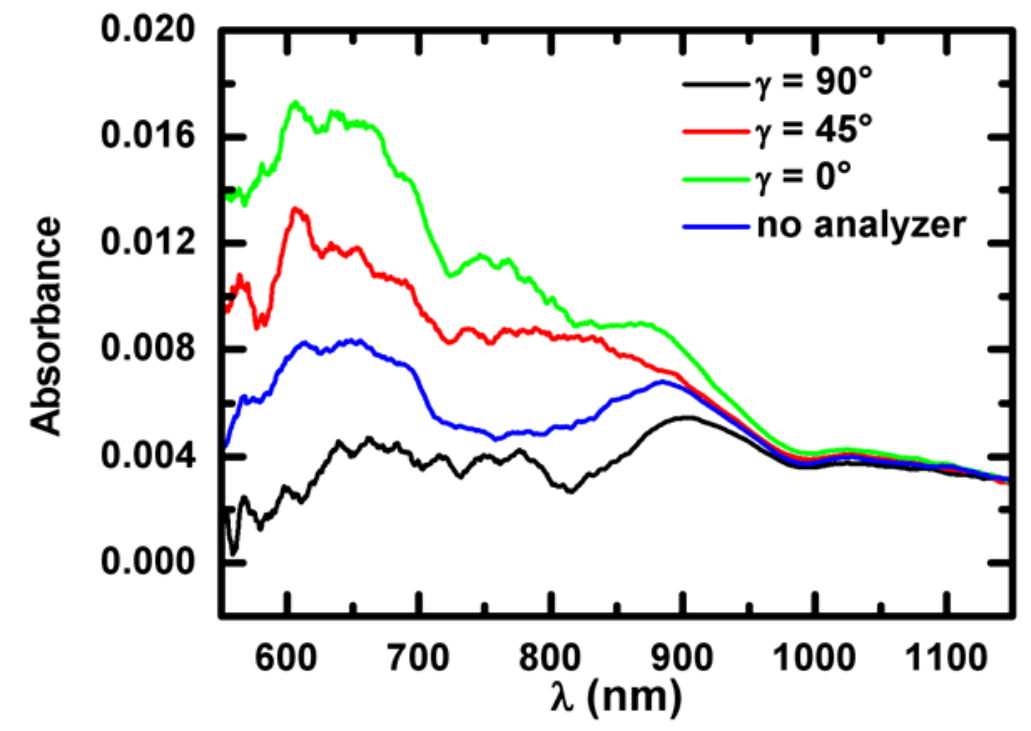

Supplementary Figure 4. Polarization dependent absorption spectra of length separated SWNTs in $1 \mathrm{w} \%$ SDBS, deposited with $v=80 \mathrm{MHz}$ and $V_{\mathrm{pp}}=40 \mathrm{~V}$. The raw data was smoothed and offset for better comparison. As reported earlier, the amount of deposited nanotubes reduces significantly at high frequencies, resulting in a very low signal intensity and a relatively big measurement error. 


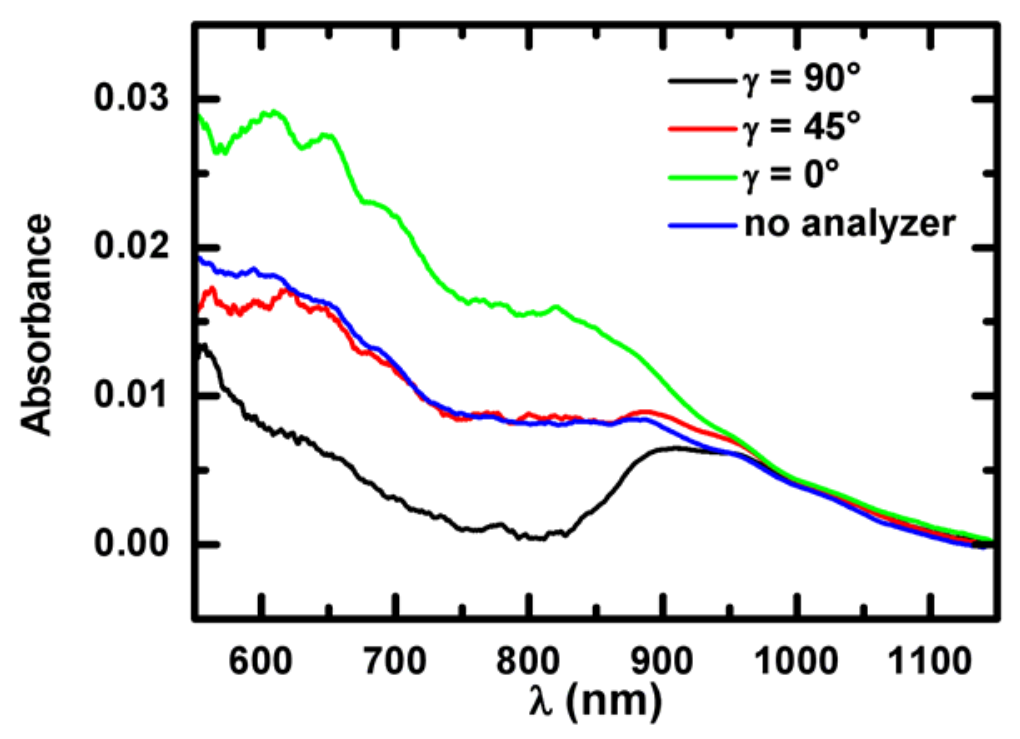

Supplementary Figure 5. Polarization dependent absorption spectra of length separated SWNTs in $1 \mathrm{w} \%$ SDBS, deposited with $v=80 \mathrm{MHz}$ and $V_{\mathrm{pp}}=80 \mathrm{~V}$. Raw data was smoothed and offset for better comparison. 


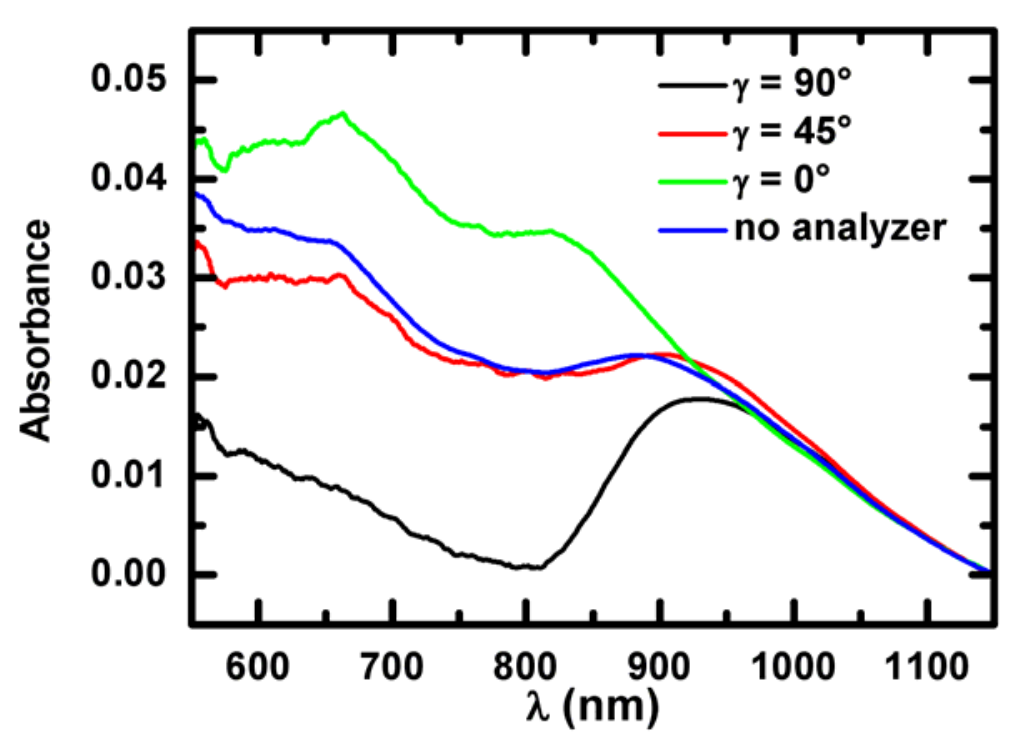

Supplementary Figure 6. Polarization dependent absorption spectra of centrifuged SWNTs in $1 \mathrm{w} \%$ SDBS, deposited with $v=80 \mathrm{MHz}$ and $V_{\mathrm{pp}}=80 \mathrm{~V}$. Raw data was smoothed and offset for better comparison. 

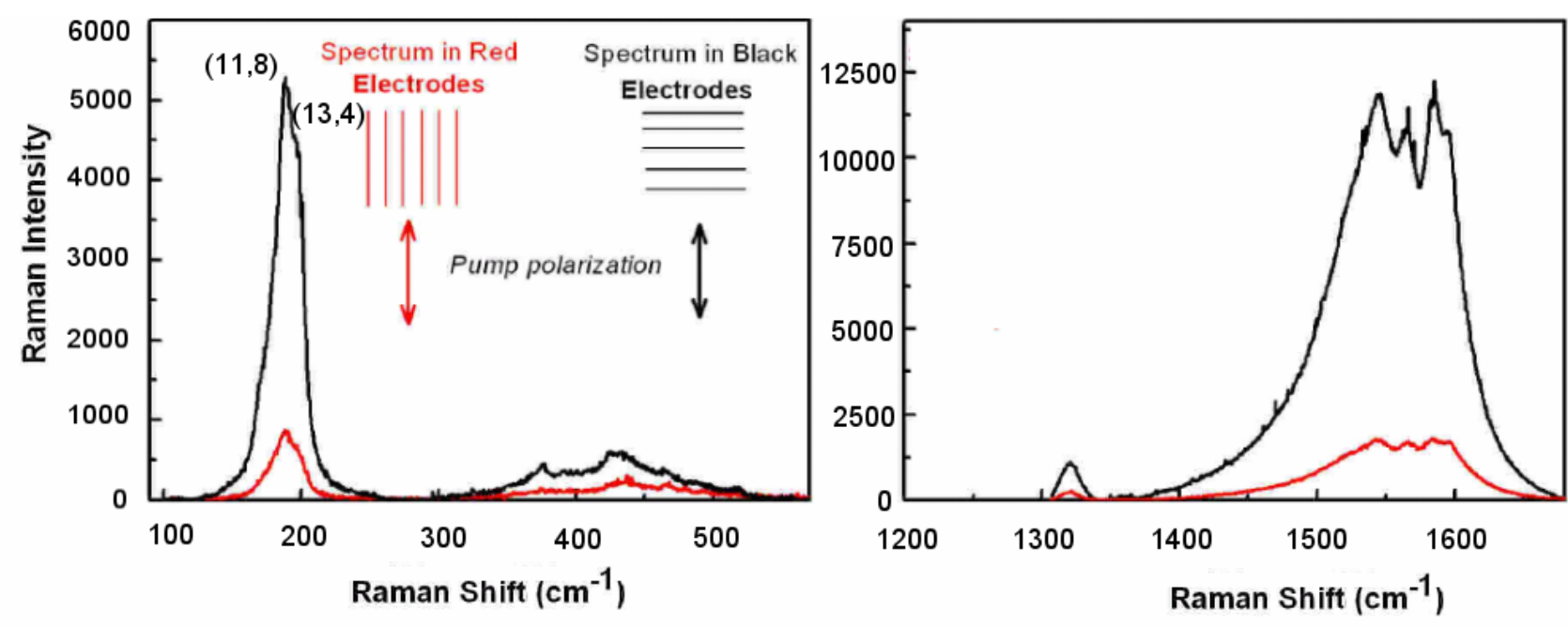

Supplementary Figure 7: Raman spectra measured with the $647.1 \mathrm{~nm}$ excitation line between the electrodes. At this wavelength primarily metallic SWNTs are excited. The intensity of the broad $\mathrm{G}^{-}$ mode (right plot) and the radial breathing mode (left plot) both decrease significantly, as the polarization of the incident light is changed from parallel to the electric field (black curves) to orthogonal to the electric field (red curves). This data is in agreement with the absorption measurements and shows that metallic SWNTs are well aligned in our samples. 

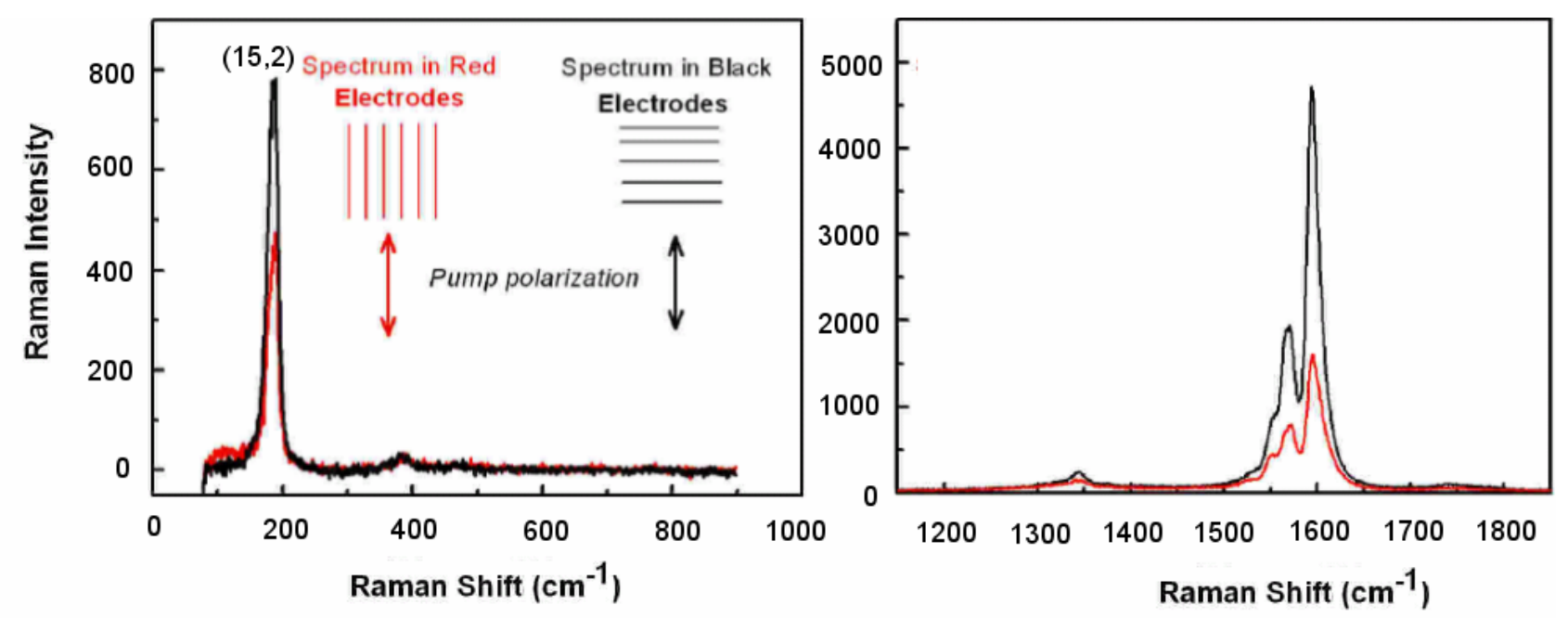

Supplementary Figure 8: Raman spectra measured with the $514.5 \mathrm{~nm}$ excitation line between the electrodes. With this wavelength primarily semiconducting SWNTs with a diameter of $\sim 1.2 \mathrm{~nm}$ are excited, which in the absorption spectrum in Figure 4 contribute to the absorbance around $880 \mathrm{~nm}$. These semiconducting SWNTs show a weak alignment, which also manifests here in the polarization dependence of the intensities of the sharp $\mathrm{G}^{+}$mode (right plot) and the radial breathing mode (left plot). However, the decrease in intensity is far less pronounced than in Supplementary Figure 7. 